\title{
Validação do instrumento de avaliação da atenção primária à saúde: PCATool-Brasil adultos
}

\author{
Validation of the Primary Care Assessment Tool: PCATool-Brazil for adults
Validación del instrumento para la evaluación de la atención primaria de salud: PCATool-Brasil adultos

Erno Harzheim. Universidade Federal do Rio Grande do Sul (UFRGS). Porto Alegre, RS, Brasil. ernoharz@terra.com.br (Autor correspondente) Mônica Maria Celestina de Oliveira. Universidade Federal de Ciências da Saúde de Porto Alegre (UFCSPA). Porto Alegre, RS, Brasil. olivmonica@gmail.com Milena Rodrigues Agostinho. Universidade Federal do Rio Grande do Sul (UFRGS). Porto Alegre, RS, Brasil. milena.rodrigues.agostinho@gmail.com Lisiane Hauser. Universidade Federal do Rio Grande do Sul (UFRGS). Porto Alegre, RS, Brasil. lisiane.hauser@ymail.com Airton Tetelbom Stein. Universidade Federal de Ciências da Saúde de Porto Alegre (UFCSPA). Porto Alegre, RS, Brasil. airton.stein@gmail.com Marcelo Rodrigues Gonçalves. Universidade Federal do Rio Grande do Sul (UFRGS). Porto Alegre, RS, Brasil. marcelorog@gmail.com Thiago Gomes da Trindade. Universidade Federal do Rio Grande do Norte (UFRN). Natal, RN, Brasil. thiagogtrindade@gmail.com Silvina Berra. Universidad Nacional de Córdoba (UNC). Córdoba, Argentina. silvinaberra@gmail.com Bruce Bartholow Duncan. Universidade Federal do Rio Grande do Sul (UFRGS). Porto Alegre, RS, Brasil. bbduncan@ufrgs.br Barbara Starfield. Department of Health Policy and Management. John Hopkins University - In memorian.

\section{Resumo}

Objetivo: traduzir e adaptar o PCATool versão usuários adultos ao contexto brasileiro e avaliar sua validade e fidedignidade. Métodos: Foi realizado um estudo transversal no qual foram entrevistados 2.404 usuários adultos residentes nas áreas adscritas da rede pública dos serviços de Atenção Primária à Saúde (APS) em Porto Alegre e usuários vinculados a um plano de autogestão. 0 processo de validação constituiu-se de tradução e tradução reversa, adaptação, validade de conteúdo, validade fatorial e fidedignidade. Resultados: Na análise fatorial, foram considerados 12 fatores que explicaram 50,83\% da variação total. Assim, foram reconhecidos os seguintes atributos da APS: acesso - acessibilidade e utilização, Iongitudinalidade, coordenação - coordenação do cuidado e sistemas de informação, integralidade - serviços disponíveis e serviços prestados, enfoque familiar e orientação comunitária. Para a consistência interna, foi obtido alfa de Cronbach variando de 0,15 a 0,90 . A estabilidade temporal não foi verificada para coordenação do cuidado $(p<0,05)$, coordenação de sistema de informação $(p<0,05)$ e integralidade de serviços prestados $(p<0,05)$. Na razão êxito da escala, todos os componentes/atributos apresentaram valores próximos de 100\%, à exceção do componente sistema de informação. Conclusões: os resultados indicaram que o PCATool-Brasil possui adequada validade e fidedignidade, podendo constituir-se em instrumento nacional de avaliação da APS para usuários adultos.

\section{Abstract}

Objective: to translate and adapt the PCATool version for adult users to the Brazilian context and assess its validity and reliability. Methods: We conducted a cross-sectional study of 2404 adult users living in areas covered from public services Primary Care in Porto Alegre, and users of a selfmanagement plan. We performed translation, reverse translation and adaptation of the instrument as well as its assessment in respect of content validity, factorial validity and reliability. Results: In the factor analysis were considered 12 factors that explained $50.83 \%$ of the total variation. In this way were recognized the attributes of Primary Care: access - accessibility and utilization; Iongitudinality; coordination - coordination of care and systems of information; comprehensiveness - services available and services provided; family orientation; and community orientation. In terms of internal consistency the values of Cronbach's alpha ranged from 0.15 to 0.90 . The stability over time was not observed for coordination of care $(p<0.05)$, coordination information of system $(p<0.05)$ and comprehensiveness of services provided $(p<0.05)$. The scaling success rate was close to $100 \%$ for all attributes, except to coordination of information system. Conclusions: the results indicated that the PCATool-Brazil has adequate validity and reliability, and it can act as a national instrument for the assessment of Primary Care from the perspective of adult users.
Como citar: Harzheim E, Oliveira MMC, Agostinho MR, Hauser L, Stein AT, Gonçalves MR et al. Validação do instrumento de avaliação da atenção primária à saúde: PCATool-Brasil adultos. Rev Bras Med Fam Comunidade. 2013; 8(29):274-84. Disponível em: http://dx.doi.org/10.5712/rbmfc8(29)829
Palavras-chave:

Validação

Atenção Primária à Saúde

Pesquisa sobre Serviços de Saúde

Keywords:

Validation

Primary Health Care Health Services Research 


\section{Resumen}

Objetivo: traducir y adaptar el PCATool versión usuarios adultos al contexto brasileño y evaluar su validez y fiabilidad. Métodos: Se realizó un estudio transversal en el cual se encuestaron a 2404 usuarios adultos residentes en áreas adscritas a la red pública de servicios de Atención Primaria de Salud (APS) en Porto Alegre y a otros usuarios vinculados a un seguro de salud de auto-gestión. El proceso de validación consistió en traducción y traducción reversa, adaptación, validez de contenido, validez factorial y fiabilidad. Resultados: En el análisis factorial se consideraron 12 factores que explicaron 50,83\% de la variación total. Así, se reconocieron los siguientes atributos de la APS: acceso - accesibilidad y utilización, longitudinalidad, coordinación - coordinación del cuidado y sistemas de información, integralidad - servicios disponibles y servicios prestados, enfoque familiar y orientación comunitaria. Para la consistencia interna se obtuvo un alfa de Cronbach oscilando entre 0,15 y 0,90. La estabilidad temporal no se verificó para la coordinación del cuidado $(p<0,05)$, la coordinación del sistema de información $(p<0,05)$ y la integralidad de servicios prestados $(p<0,05)$. En la razón de éxito de la escala todos los componentes/atributos presentaron valores próximos al 100\%, con excepción del componente sistema de información. Conclusiones: Ios resultados indicaron que el PCATool-Brasil tiene una adecuada validez y fiabilidad, pudiendo constituirse en herramienta nacional para la evaluación de la APS en usuarios adultos.
Palabras clave:

Validación

Atención Primaria de Salud Investigación sobre Servicios de Salud

\section{Introdução}

Em busca da construção de um sistema nacional de saúde e do fortalecimento dos princípios que norteiam o Sistema Único de Saúde (SUS), o Ministério da Saúde brasileiro baseou-se em experiências prévias nacionais e de países como Inglaterra, Canadá, Espanha e Cuba para implementar mudanças na assistência ambulatorial nacional. Esses países organizaram seus sistemas de saúde priorizando o acesso universal com cuidado continuado, integral e coordenado, junto às comunidades e seu contexto social, ou seja, desenvolveram sistemas com forte orientação para a Atenção Primária à Saúde (APS). No Brasil, o alicerce da mudança assistencial iniciou na década de 70, com experiências isoladas em Niterói, Porto Alegre e outros locais, e foi consolidar-se apenas em meados dos anos 90, com a implantação do Programa de Agentes Comunitários de Saúde ${ }^{1}$ em 1991 e, posteriormente, com a criação da Estratégia Saúde da Família (ESF)². Essa estratégia foi adotada como modelo de expansão e reorganização da APS e vem crescendo em número de equipes e cobertura populacional.

Starfield ${ }^{3}$ conceituou a APS por meio de seus atributos essenciais (acesso de primeiro contato, longitudinalidade e integralidade da atençáo, além da coordenaçáo da assistência) e derivados (atenção centrada na família, orientação comunitária e competência cultural dos profissionais). Esta operacionalizaçáo do conceito permite identificar o grau de orientaçáo à APS, que propicia a comparabilidade entre sistemas ou tipos de serviços, além da associação entre a presença dos atributos e a efetividade da atenção, tanto em nível individual, quanto populacional. Nesse sentido, Starfield et al. ${ }^{4,5}$ criaram e validaram um instrumento nos Estados Unidos da América, o Primary Care Assessment Tool (PCATool) que mede a presença e a extensão dos atributos da APS para usuários adultos e para crianças. No Brasil, em 2006, foi realizada a primeira validaçáo do PCATool versão usuários crianças ${ }^{6,7}$. Outras iniciativas de validação ou uso desse instrumento vêm sendo realizadas em diversos países, como Taiwan ${ }^{8,9}$, Hong Kong ${ }^{10}$, Canadá ${ }^{11,12}$ e Espanha ${ }^{13}$.

Num momento de forte expansão da APS nas Américas e, particularmente, no Brasil, torna-se imprescindível uma produção rigorosa de conhecimento que possibilite avaliar a qualidade dos cuidados primários prestados à populaçáo, principalmente no que tange às características da estrutura, processo de atenção e desfechos em saúde. Associado a este fato, as mudanças no padrão de morbimortalidade nos países em desenvolvimento, com a diminuição acentuada das doenças infectoparasitárias e a forte escalada das doenças crônicas não transmissíveis e de causas externas ${ }^{14,15}$, têm imposto novos desafios à organização dos serviços de saúde, com a APS ocupando o centro de comunicação de redes integradas de serviços de saúde ${ }^{16}$.

No Brasil, foram realizados diversos estudos de avaliação da ESF comparando-a ao modelo tradicional ainda vigente em nosso país ${ }^{17,18}$. Entretanto, a falta de uniformidade conceitual utilizada nas diversas definiçóes de APS e a grande variabilidade metodológica desses e de outros estudos, não nos permite estabelecer uma visão homogênea do processo de reorganização dos serviços de APS. Devido à necessidade de evoluir na produção de métodos rigorosos de avaliação da APS, o objetivo deste estudo foi traduzir e adaptar o PCATool versão usuários adultos ao contexto brasileiro, assim como avaliar sua validade e fidedignidade. 


\section{Métodos}

\section{O PCATool}

Esse instrumento ${ }^{19}$ é embasado no marco teórico da APS e foi desenvolvido com o objetivo de medir a presença e extensão dos atributos essenciais e derivados da APS $^{3}$ além do grau de afiliação do usuário ao serviço de saúde. Originalmente, o instrumento versão usuários adultos contém 92 itens divididos entre sete atributos da APS com escala Likert variando de ' 1 = com certeza náo' a ' 4 = com certeza sim'. A partir da média das respostas dos itens que constituem o atributo, é possível obter-se um escore para cada atributo e também o Escore Essencial e o Escore Geral de APS.

O Escore Essencial é obtido pela média entre os componentes dos atributos essenciais (primeiro contato, longitudinalidade, coordenação e integralidade) e o grau de afiliaçáo do usuário com o serviço. O Escore Geral é obtido pela média entre os componentes dos atributos essenciais, dos atributos derivados e do grau de afiliaçáo do usuário com o serviço. Alguns atributos sáo formados por mais de um componente, por exemplo, o atributo acesso de primeiro contato é formado pelo componente 'acessibilidade' e pelo componente 'utilização'. Os valores dos escores são padronizados para uma escala variando de 0 a 10, sendo os valores iguais ou superiores a 6,6 considerados como Alto Escore, o que corresponde a respostas para as opçóes 3 ou 4 na escala original do instrumento. A padronização para a escala 0-10 é realizada da seguinte forma:

$$
\text { EscorePadronizado }=\frac{(\text { Escore }-1)}{(4-1)} \times 10
$$

\section{Delineamento e amostra}

Estudo transversal de base populacional representativo da população adscrita aos serviços de APS do município de Porto Alegre, Rio Grande do Sul, Brasil, realizado entre 2006 e 2007. O protocolo geral deste estudo foi publicado anteriormente ${ }^{20}$.

A amostra foi composta por indivíduos adultos (18 anos ou mais) que residiam há pelo menos um ano em áreas de adscrição dos quatro serviços da rede pública de APS do município (Estratégia Saúde da Família - ESF, Unidade Básica de Saúde - UBS, Serviço de Saúde Comunitária do Grupo Hospitalar Conceição-SSC-GHC e Centro de Saúde Escola Murialdo - CSEM) ou eram associados há pelo menos um ano na Caixa de Assistência dos Funcionários do Banco do Brasil - CASSI, vinculados à estratégia de APS deste plano de autogestão. O plano amostral complexo descrito detalhadamente no protocolo de estudo ${ }^{20}$ foi considerado no processo de amostragem, mas, a fim de atingir os objetivos da validação, foi estimado um tamanho de amostra igual a 410 indivíduos, o que representa um mínimo de 5 respondentes para cada item do instrumento ${ }^{21}$. Foram excluídos os indivíduos que não apresentavam condição física/mental para responder ao questionário, ou com última consulta em um serviço de saúde de Porto Alegre antes de janeiro de 1996 (ano de municipalização da saúde na cidade) ou que utilizavam como referência serviço de saúde em outro município. As entrevistas foram realizadas nos domicílios dos indivíduos por profissionais e estudantes da área da saúde devidamente treinados.

\section{Tradução e adaptação}

A traduçáo foi realizada em duas etapas, do inglês para o português e vice-versa, por uma tradutora nativa da língua inglesa. A versão original foi comparada pela equipe de investigação com a versão em inglês resultante da traduçáo reversa, para correção das possíveis falhas neste processo. $\mathrm{Na}$ adaptação, foram consideradas características culturais da população e de organização dos serviços de saúde.

No processo de tradução e adaptação, foram consideradas as propriedades de equivalência semântica, prática e cultural do instrumento. Para tanto, se entrevistaram, consecutivamente, seis indivíduos, homens e mulheres usuários de serviços de APS, entre 22 e 66 anos. Foram realizadas entrevistas cognitivas a fim de comprovar a compreensão e a interpretação correta do significado das perguntas, pela qual a pessoa deveria ser capaz, durante as entrevistas, de reformular a pergunta ou falar sobre seu conteúdo com outras palavras, mantendo o mesmo significado. A cada entrevista, o questionário era modificado a fim de incorporar as sugestôes que facilitavam a compreensão. Posteriormente, 32 questionários foram aplicados, sendo discutidas e corrigidas as dificuldades de compreensão identificadas. 


\section{Validade e fidedignidade}

- Validade de conteúdo

$\mathrm{Na}$ validade de conteúdo, foi verificado se o instrumento considerou todos os aspectos relevantes do conceito em estudo, de modo que cada pergunta proporcionasse um dado observável acerca do conceito do atributo para o qual foi formulado.

- Validade fatorial

Utilizou-se a análise fatorial com extração via componentes principais e método de rotação Varimax. Foram selecionados os fatores com três ou mais itens com carga fatorial superior a $0,35^{21}$. Cada fator foi avaliado conceitualmente, junto à autora principal do instrumento original, a fim de evitar que qualquer fator não representasse conceitualmente um atributo da APS.

- Fidedignidade

A fidedignidade deste instrumento foi observada utilizando a consistência interna, a estabilidade no tempo e a razão êxito da escala. Para avaliar a consistência interna de cada componente, utilizou-se a correlação item-total, considerando adequados os itens com valor superior a $0,30^{21}$ e o coeficiente alfa de Cronbach, idealmente igual ou superior a $0,70^{21}$.

$\mathrm{Na}$ estabilidade no tempo, $10 \%$ dos usuários da amostra foram reentrevistados (reteste), permitindo que os escores produzidos pela coleta de dados na entrevista (teste) fossem comparados aos escores obtidos no reteste, utilizando o teste de Wilcoxon para amostras emparelhadas. A relação teste-reteste também foi verificada pela correlação intraclasse. Na razão êxito da escala, verifica-se o quociente entre o número de vezes que correlaçôes entre os itens de um atributo foram superiores às correlaçóes de cada um desses itens com os outros atributos e o total de correlaçôes efetuadas nesse atributo. Valores elevados dessa medida sugerem maior discriminação do atributo.

\section{Aspectos éticos}

O projeto "Avaliação da qualidade do processo de atenção e da sua efetividade sobre a saúde do adulto no Programa Saúde da Família e modelos alternativos no município de Porto Alegre" foi aprovado no comitê de ética da Secretaria Municipal da Saúde, do Centro de Saúde Escola Murialdo, do Grupo Hospitalar Conceição, do Grupo de Pesquisa e Pós-Graduação do Hospital de Clínicas de Porto Alegre, da Caixa de Assistência dos Funcionários do Banco do Brasil - Regional RS e da Universidade Federal do Rio Grande do Sul. As entrevistas com os usuários foram realizadas mediante leitura e assinatura do Termo de Consentimento Livre e Esclarecido (TCLE).

\section{Resultados}

\section{Característica da amostra}

A amostra total da pesquisa foi de 3.014 indivíduos. Entre esses foram selecionados os 2.404 indivíduos que constituem a amostra deste estudo, porque referiram como serviço de saúde preferencial, um serviço ou profissional que representava um provedor de APS.

O serviço preferencial para 24\% dos indivíduos foi a ESF; para 12,5\%, a UBS Tradicional; para 7,8\%, o CSEM; para 5,6\%, o SSC-GHC; para 8,0\%, a CASSI; para 12,5\%, ambulatório do plano de saúde; para 24,1\%, consultórios médicos; para 2,6\%, ambulatórios de empresas; e para 2,8\%, clínicas ou atendimento domiciliar.

A idade média desses indivíduos foi de 46,5 anos (desvio padrão=17,3 anos). Aproximadamente $63 \%$ dos indivíduos foram do sexo feminino e quase $69 \%$ declararam cor da pele branca. A escolaridade média foi de 9,6 anos (desvio padrão=4,9 anos). Conforme o critério padrão de classificação econômica Brasil/2008 da Associação Brasileira das Empresas de Pesquisa (ABEP), 1,2\% dos indivíduos pertencia à classe $\mathrm{E} ; 10,6 \%$ à classe $\mathrm{D} ; 43,8 \%$ à classe $\mathrm{C} ; 37 \%$ à classe $\mathrm{B} ; \mathrm{e} 7,5 \%$ à classe $\mathrm{A}$. 


\section{Tradução e adaptação}

$\mathrm{Na}$ comparação entre a versão original e a versão resultante da tradução reversa não foram encontradas diferenças de conteúdo ou significado. A adaptação foi realizada segundo características culturais do Brasil. Nessa etapa, cinco perguntas foram excluídas e algumas modificadas. Também foi realizado um piloto, considerando uma amostra semelhante à população deste estudo, que possibilitou o esclarecimento de outras perguntas dúbias e a finalização do instrumento com as correçôes necessárias. Posteriormente, o instrumento foi aplicado a seis indivíduos quando se avaliou o grau de entendimento para cada uma das perguntas até o esgotamento de dúvidas, novamente adaptando-se o texto de algumas perguntas.

\section{Validade de conteúdo}

Foi considerada a adequaçáo com que uma medida afere o componente a que se refere, utilizando a avaliaçáo conceitual dos fatores. Esse processo foi realizado com a participação da autora principal do instrumento original (BS).

\section{Validade fatorial}

Os itens mostraram-se distribuídos de forma mais homogênea nos fatores que representam conceitualmente os componentes da APS quando realizada análise fatorial considerando 12 fatores, com variação total explicada de 50,83\%. Os itens do acesso foram captados pelos fatores um e oito. A longitudinalidade mostrou-se presente no fator dois e no fator dez. Os itens de orientação comunitária foram captados em sua totalidade no fator três, assim como os itens de coordenação do cuidado no fator sete e de acesso-utilização no fator doze. A integralidade de serviços prestados teve seus itens distribuídos nos fatores quatro, cinco e seis, e a integralidade de serviços disponíveis apresentou itens no fator nove e no fator onze. Os itens de enfoque familiar foram captados no fator cinco, no qual também foram captados alguns itens de integralidade de serviços prestados. As cargas fatoriais são apresentadas na Tabela 1.

Tabela 1. Cargas fatoriais para a validade fatorial, correlação item-total e escore médio dos itens. Porto alegre, Rio Grande do Sul, Brasil, 2007.

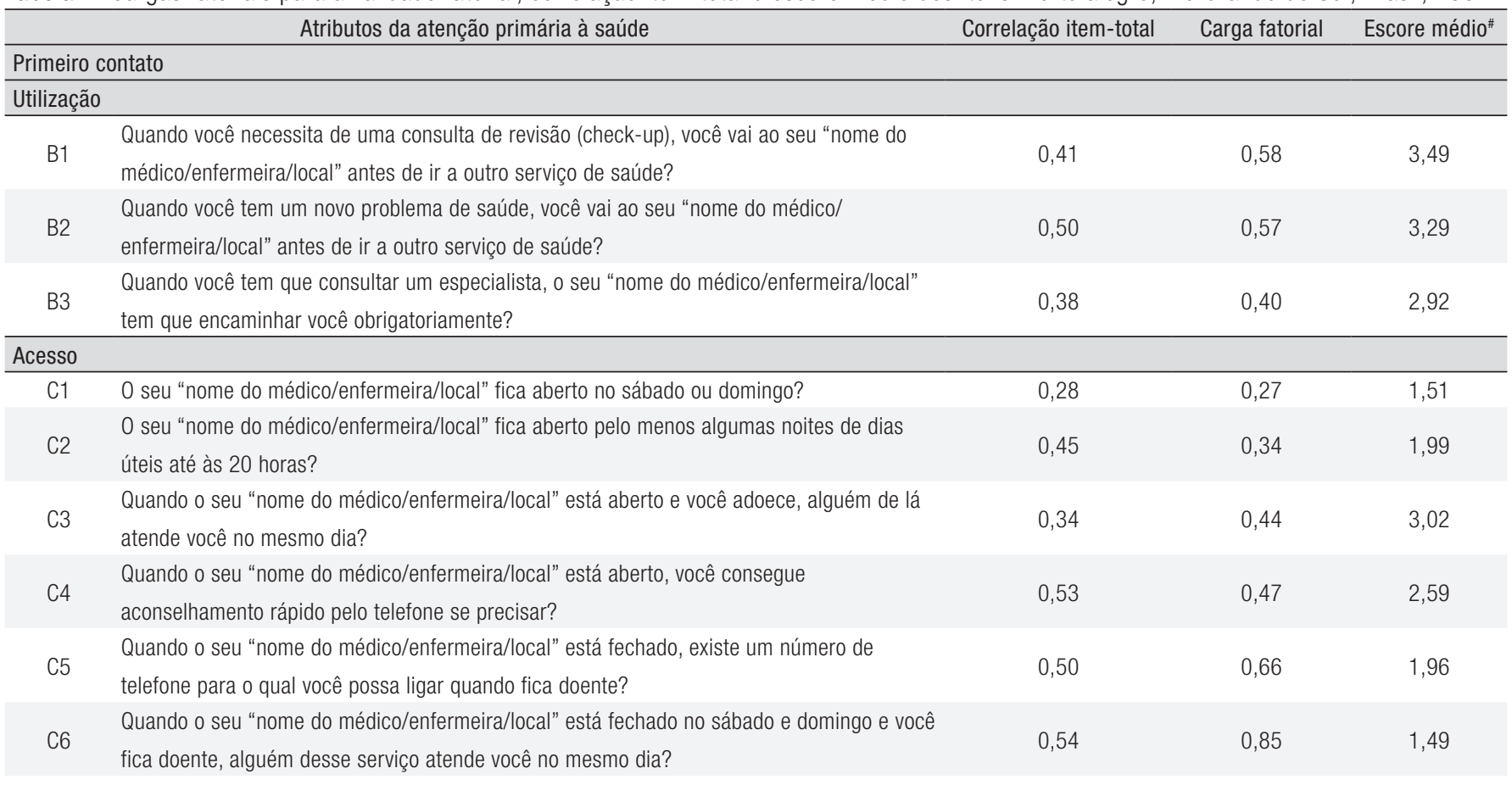


Tabela 1. Cargas fatoriais para a validade fatorial, correlação item-total e escore médio dos itens. Porto alegre, Rio Grande do Sul, Brasil, 2007.

Continuação

\section{Atributos da atenção primária à saúde}

Correlação item-total Carga fatorial Escore médio\#

C7 Quando o seu "nome do médico/enfermeira/local" está fechado e você fica doente durante a

Correlaçăo item-total Carga fatorial Escore médio

noite, alguém desse serviço atende você naquela noite?

C8

É fácil marcar hora para uma consulta de revisão (check-up) nesse "nome do médico/

enfermeira/local"?

0,55

0,58

Uma vez que você chega ao seu "nome do médico/enfermeira/local", você tem que esperar

C9 mais de 30 minutos para consultar com o médico ou enfermeira (sem contar triagem ou acolhimento)?

Você tem que esperar por muito tempo ou falar com muitas pessoas para marcar hora no

C10 seu "nome do médico/enfermeira/local"?

E11 É difícil para você conseguir atendimento médico do seu "nome do médico/enfermeira/local"

C11 quando pensa que é necessário?

C12

Quando você tem que ir ao seu "nome do médico/enfermeira/local", você tem que faltar ao trabalho ou à escola para ir ao serviço de saúde?

\section{Longitudinalidade}

Quando você vai ao seu "nome do médico/enfermeira/local", é o mesmo médico ou enfermeira que atende você todas as vezes?

Você acha que o seu "nome do médico/enfermeira/local " entende o que você diz ou

$\mathrm{D} 2$ pergunta?

D3 0 seu "nome do médico/enfermeira/local" responde a suas perguntas de maneira que você entenda?

Se você tiver uma pergunta, pode telefonar e falar com o médico ou enfermeira que melhor

D4 conhece você?

D5

0 seu "nome do médico/enfermeira/local" Ihe dá tempo suficiente para falar sobre as suas preocupações ou problemas?

Você se sente à vontade contando as suas preocupações ou problemas ao "nome do

D6 médico/enfermeira/local"?

D7 0 seu "nome do médico/enfermeira/local" conhece você mais como pessoa do que somente como alguém com um problema de saúde?

D8 0 seu "nome do médico/enfermeira/local" sabe quem mora com você?

9 "O seu nome do médico/enfermeira/local" sabe quais problemas são mais importantes para

D9 você?

D10 0 seu "nome do médico/enfermeira/local" conhece a sua história médica completa?

D11 0 seu "nome do médico/enfermeira/local" sabe a respeito do seu trabalho ou emprego?

0 seu "nome do médico/enfermeira/local" saberia de alguma forma se você tivesse

D12 problemas em obter ou pagar por medicamentos de que você precisa?

D13 0 seu "nome do médico/enfermeira/local" sabe a respeito de todos os medicamentos que você está tomando?

D14 Você mudaria do "nome do médico/enfermeira/local" para outro serviço de saúde se isto fosse muito fácil de fazer?

Coordenação

Cuidado

E2 0 seu "nome do médico/enfermeira/local" sugeriu que você fosse consultar com este especialista ou ao serviço especializado?

0 seu "nome do médico/enfermeira/local" sabe que você fez essas consultas com este

E3 especialista ou o serviço especializado?

0 seu "nome do médico/enfermeira/local" discutiu com você diferentes serviços onde você

E4 poderia ser atendido para este problema de saúde?

0 seu "nome do médico/enfermeira/local" ou alguém que trabalha no/com "nome do

E5 médico/enfermeira/local" ajudou-o a marcar esta consulta?

0,49
0,54

0,60

0,35

0,43

0,47

0,44

0,53

0,51

0,52

0,51

0,47

0,64

0,59

0,60

0,54

0,55

0,49

(

0,59

0,85

0,73

1,47

2,99

0,59

0,64

3,05

2,89

2,98

3,64 3,50

5,52 3,04

2,70 
Tabela 1. Cargas fatoriais para a validade fatorial, correlação item-total e escore médio dos itens. Porto alegre, Rio Grande do Sul, Brasil, 2007.

Continuação

\begin{tabular}{|c|c|c|c|c|}
\hline & Atributos da atenção primária à saúde & Correlação item-total & Carga fatorial & Escore médio\# \\
\hline E6 & $\begin{array}{l}0 \text { seu "nome do médico/enfermeira/local" escreveu alguma informação para o especialista a } \\
\text { respeito do motivo desta consulta? }\end{array}$ & 0,54 & 0,56 & 2,11 \\
\hline E7 & 0 seu "nome do médico/enfermeira/local" sabe quais foram os resultados desta consulta? & 0,58 & 0,76 & 2,63 \\
\hline E8 & $\begin{array}{l}\text { Depois que você foi a este especialista ou ao serviço especializado, o seu "nome do médico/ } \\
\text { enfermeira/local" conversou com você sobre o que aconteceu durante esta consulta? }\end{array}$ & 0,56 & 0,73 & 2,05 \\
\hline
\end{tabular}
0 seu "nome do médico/enfermeira/local" pareceu interessado na qualidade do cuidado que

E9 Ine foi dado, isto é, lhe pergunta se você foi bem ou mal atendido por este especialista ou serviço especializado?

\begin{tabular}{|c|c|c|c|c|}
\hline \multicolumn{5}{|c|}{ Sistema de Informação } \\
\hline F1 & $\begin{array}{l}\text { Quando você vai ao "nome do médico/enfermeira/local", você leva algum de seus registros } \\
\text { médicos ou boletins de atendimento que recebeu no passado? }\end{array}$ & 0,04 & 0,23 & 1,97 \\
\hline F2 & Você poderia ler o seu prontuário/ficha se quisesse no "nome do médico/enfermeira/local"? & 0,10 & 0,35 & 2,72 \\
\hline F3 & $\begin{array}{l}\text { Quando você vai ao "nome do médico/enfermeira/local", seu prontuário/ficha está sempre } \\
\text { disponível na consulta? }\end{array}$ & 0,13 & 0,20 & 3,76 \\
\hline \multicolumn{5}{|c|}{ Integralidade } \\
\hline \multicolumn{5}{|c|}{ Serviços Disponíveis } \\
\hline G1 & Respostas a perguntas sobre nutrição e dieta & 0,27 & 0,33 & 3,08 \\
\hline G2 & $\begin{array}{l}\text { Verificar se sua família pode participar de algum programa de assistência social ou } \\
\text { benefícios sociais }\end{array}$ & 0,41 & 0,43 & 2,13 \\
\hline G3 & Programa de suplementação de leite e alimentos & 0,40 & 0,41 & 1,93 \\
\hline G4 & Vacinas (imunizações) & 0,46 & 0,42 & 3,05 \\
\hline G5 & Exame dentário & 0,53 & 0,48 & 2,27 \\
\hline G6 & Tratamento dentário & 0,51 & 0,48 & 2,17 \\
\hline G7 & Planejamento familiar ou métodos anticoncepcionais & 0,49 & 0,71 & 2,96 \\
\hline G8 & $\begin{array}{l}\text { Aconselhamento ou tratamento para o uso prejudicial de drogas (lícitas ou ilícitas, ex.: } \\
\text { álcool, cocaína, remédios para dormir) }\end{array}$ & 0,52 & 0,62 & 2,50 \\
\hline G9 & Aconselhamento para problemas de saúde mental & 0,49 & 0,59 & 2,54 \\
\hline G10 & Sutura de um corte que necessite de pontos & 0,46 & 0,68 & 2,16 \\
\hline G11 & Aconselhamento e solicitação de teste anti-HIV & 0,45 & 0,59 & 2,86 \\
\hline G12 & Identificação (Algum tipo de avaliação) de problemas auditivos (para escutar) & 0,57 & 0,58 & 2,22 \\
\hline G13 & Identificação (Algum tipo de avaliação) de problemas visuais (para enxergar) & 0,53 & 0,59 & 2,21 \\
\hline G14 & Colocação de tala para tornozelo torcido & 0,44 & 0,73 & 1,90 \\
\hline G15 & Remoção de verrugas & 0,49 & 0,72 & 1,77 \\
\hline G16 & Exame preventivo para câncer de colo de útero (Teste de Papanicolau) & 0,49 & 0,73 & 2,95 \\
\hline G17 & Aconselhamento sobre como parar de fumar & 0,45 & 0,57 & 2,66 \\
\hline G18 & Cuidados pré-natais & 0,55 & 0,71 & 3,17 \\
\hline G19 & Remoção de unha encravada & 0,54 & 0,72 & 1,87 \\
\hline G20 & $\begin{array}{l}\text { Aconselhamento sobre as mudanças que acontecem com o envelhecimento (ex.: diminuição } \\
\text { da memória, risco de cair) }\end{array}$ & 0,29 & 0,31 & 2,44 \\
\hline G21 & $\begin{array}{l}\text { Orientações sobre cuidados no domicílio para alguém da sua família como curativos, troca } \\
\text { de sondas, banho na cama }\end{array}$ & 0,45 & 0,39 & 2,54 \\
\hline \multirow{3}{*}{ G22 } & 0 que fazer caso alguém de sua família fique incapacitado e não possa tomar decisões & & & \\
\hline & sobre a sua saúde (ex.: doação de órgãos, caso alguém de sua família fique incapacitado & 0,42 & 0,49 & 2,25 \\
\hline & para decidir, por exemplo, em estado de coma) & & & \\
\hline \multicolumn{5}{|c|}{ Serviços Prestados } \\
\hline $\mathrm{H} 1$ & Conselhos sobre alimentação saudável ou sobre dormir suficientemente & 0,53 & 0,65 & 2,81 \\
\hline $\mathrm{H} 2$ & Segurança no lar, como guardar medicamentos em segurança & 0,54 & 0,57 & 2,13 \\
\hline $\mathrm{H} 3$ & $\begin{array}{l}\text { Aconselhamento sobre } 0 \text { uso de cinto de segurança ou assentos seguros para crianças ao } \\
\text { andar de carro }\end{array}$ & 0,48 & 0,65 & 1,41 \\
\hline
\end{tabular}


Tabela 1. Cargas fatoriais para a validade fatorial, correlação item-total e escore médio dos itens. Porto alegre, Rio Grande do Sul, Brasil, 2007.

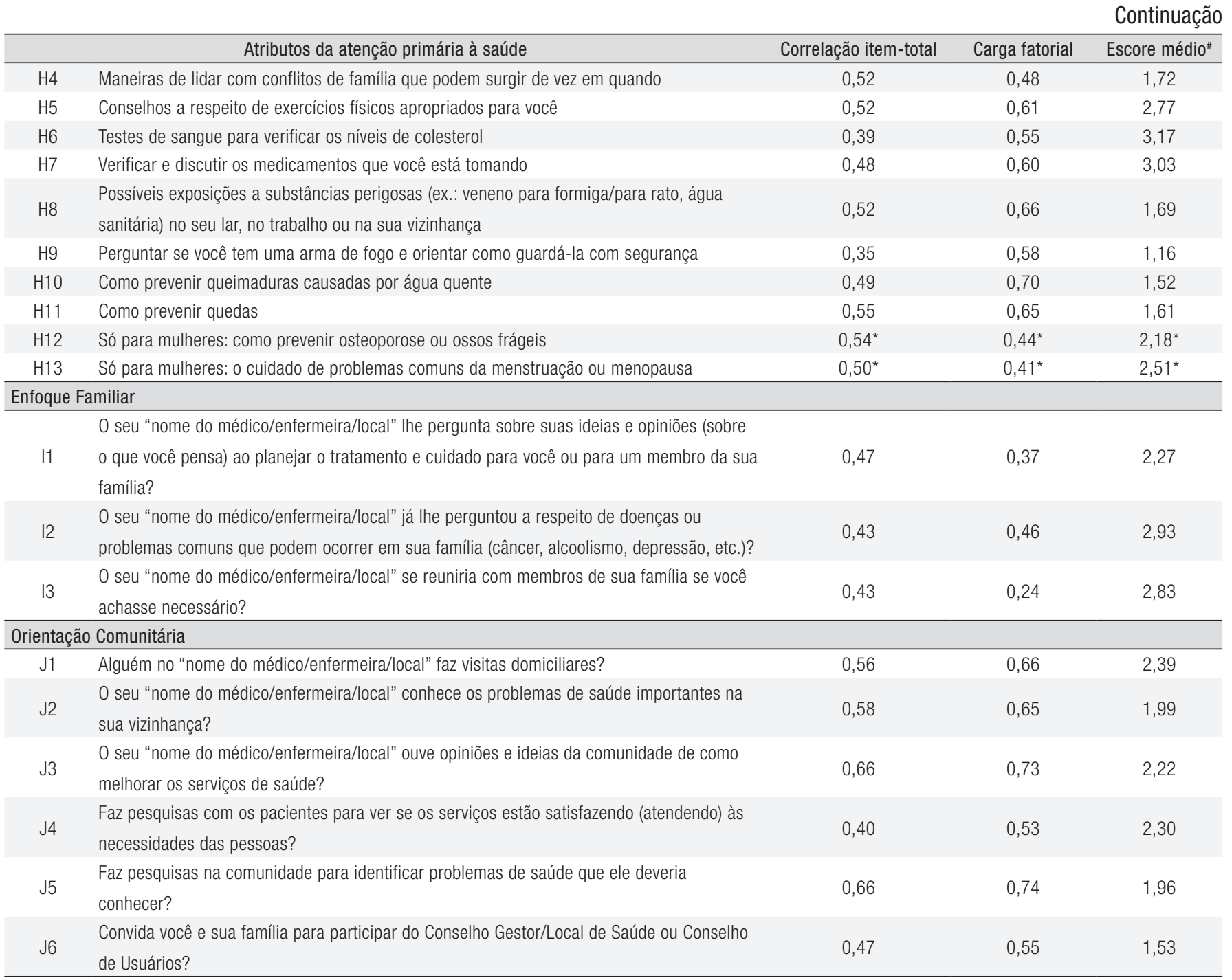

\#Escore variando de 1 a $4 .{ }^{*}$ Obtidos por meio somente da amostra de usuários do sexo feminino ( $\left.n=1484\right)$.

\section{Fidedignidade}

Para a consistência interna, considerando a correlaçâo item-total, o item sobre atendimento aos sábados e domingos foi o único no componente acesso de primeiro contato que apresentou correlação abaixo do adequado. Para o componente coordenação do sistema de informação, os três itens que constituem este atributo tiveram correlação abaixo do adequado. No componente integralidade dos serviços disponíveis, o item referente a respostas sobre nutrição e dieta e também o item de aconselhamento sobre mudanças que ocorrem no envelhecimento mostraram correlação item-total com valor inferior ao adequado. Por outro lado, para os componentes acesso-utilização, longitudinalidade, coordenação do cuidado, orientação familiar e orientação comunitária, todos os itens apresentaram correlação adequada (Tabela 1).

Para a consistência interna utilizando o alfa de Cronbach, observaram-se valores do coeficiente abaixo do adequado para o componente acesso-utilização ( $(\mathrm{llf} a=0,62)$, para o componente coordenação sistema de informaçôes $(\mathrm{alfa}=0,15)$ e orientação familiar (alfa=0,63), conforme apresentado na Tabela 2 . 
Tabela 2. Medidas de fidedignidade para os sete atributos do PCATool-Brasil. Porto Alegre, Rio Grande do Sul, Brasil, 2007.

\begin{tabular}{|c|c|c|c|c|c|}
\hline $\begin{array}{l}\text { Atributos da Atenção Primária à } \\
\text { Saúde }\end{array}$ & $\mathrm{n}$ & Alfa de Cronbach & $\begin{array}{l}\text { Razão de êxito da } \\
\text { escala }\end{array}$ & $\begin{array}{l}\text { Comparação teste- } \\
\text { reteste (valor-p) }{ }^{+}\end{array}$ & $\begin{array}{c}\text { Correlação intraclasse } \\
\text { teste-reteste }\end{array}$ \\
\hline Primeiro contato - Utilização & 2403 & 0,62 & $26 / 27(96,3 \%)$ & 0,97 & 0,69 \\
\hline Primeiro contato - Acesso & 2119 & 0,82 & $108 / 108(100 \%)$ & 0,19 & 0,67 \\
\hline Longitudinalidade & 2387 & 0,86 & $125 / 126(99,2 \%)$ & 0,06 & 0,83 \\
\hline Coordenação Sistema de Informação & 2401 & 0,15 & $12 / 27(44,4 \%)$ & $<0,01$ & 0,04 \\
\hline Integralidade - Serviços Disponíveis & 2354 & 0,88 & 192/198 (97\%) & 0,20 & 0,85 \\
\hline Integralidade - Serviços Prestados* & 2390 & 0,82 & 98/99 (98.9\%) & \multirow{2}{*}{$<0,01$} & \multirow{2}{*}{0,88} \\
\hline Integralidade - Serviços Prestados"\# & 1484 & 0,84 & $116 / 117(99,2 \%)$ & & \\
\hline Todos os Itens & 1202 & 0,90 & - & $<0,001$ & 0,96 \\
\hline
\end{tabular}

${ }^{+}$Associado ao teste Wilcoxon para amostras emparelhadas. *Itens de H1 a H11, considerando usuários de ambos os sexos. "Itens de H1 a H13, considerando somente usuários do sexo feminino.

Nos componentes coordenação do cuidado $(\mathrm{p}<0,05)$, coordenação do sistema de informaçôes $(\mathrm{p}<0,05)$ e integralidade dos serviços prestados $(\mathrm{p}<0,05)$ não foi observada a estabilidade temporal que assegura a fidedignidade do instrumento ao longo do tempo (Tabela 2). Os menores valores para a correlaçáo intraclasse foram verificados para o componente coordenação do sistema de informação $(0,04)$, para o atributo orientação comunitária $(0,4)$, e para o atributo orientação familiar $(0,4)$ (Tabela 2). Para a razão êxito da escala (REE) todos os resultados foram próximos de 100\%, à exceção do componente coordenação sistema de informação (REE=44\% - Tabela 2).

O número de itens e o coeficiente alfa de Cronbach para o PCATool versão usuários adultos nos EUA e também no Brasil, são mostrados na Tabela 3. A versão brasileira contempla o atributo orientação familiar e maior número de itens nos componentes acesso de primeiro contato - acessibilidade e integralidade - serviços disponíveis e no atributo orientação comunitária. No entanto, na versão brasileira, observa-se menor número de itens para o atributo longitudinalidade. Os valores e alfa de Cronbach para ambos os processos de validação foram semelhantes.

$\mathrm{Na}$ Tabela 4, verifica-se que a variância explicada acumulada pelos fatores definidos alcança o valor de 50,16\%.

Tabela 3. Número de itens e alfa de Cronbach PCATool EUA (original) e PCATool-Brasil. Porto Alegre, Rio Grande do Sul, Brasil, 2007.

\begin{tabular}{|c|c|c|c|c|c|c|}
\hline $\begin{array}{c}\text { Atributos da atenção primária à } \\
\text { saúde }\end{array}$ & \multicolumn{3}{|c|}{ PCATool EUA $(n=1000)$} & \multicolumn{3}{|c|}{ PCATool Brasil $(n=2404)$} \\
\hline Primeiro contato - Utilização & 3 & 0,64 & 888 & 3 & 0,62 & 2403 \\
\hline Primeiro contato - Acesso & 4 & 0,71 & 892 & 12 & 0,82 & 2119 \\
\hline Coordenação cuidado & 8 & 0,88 & 483 & 8 & 0,80 & 1419 \\
\hline Coordenação Sistema de Informação & & & & 3 & 0,15 & 2401 \\
\hline Integralidade - Serviços Disponíveis & 21 & 0,95 & 887 & 22 & 0,88 & 2354 \\
\hline Integralidade - Serviços Prestados & 13 & 0,93 & 875 & 13 & 0,82 & 1458 \\
\hline
\end{tabular}


Tabela 4. Distribuição dos itens do PCATool e variação explicada nos fatores provenientes da análise fatorial.

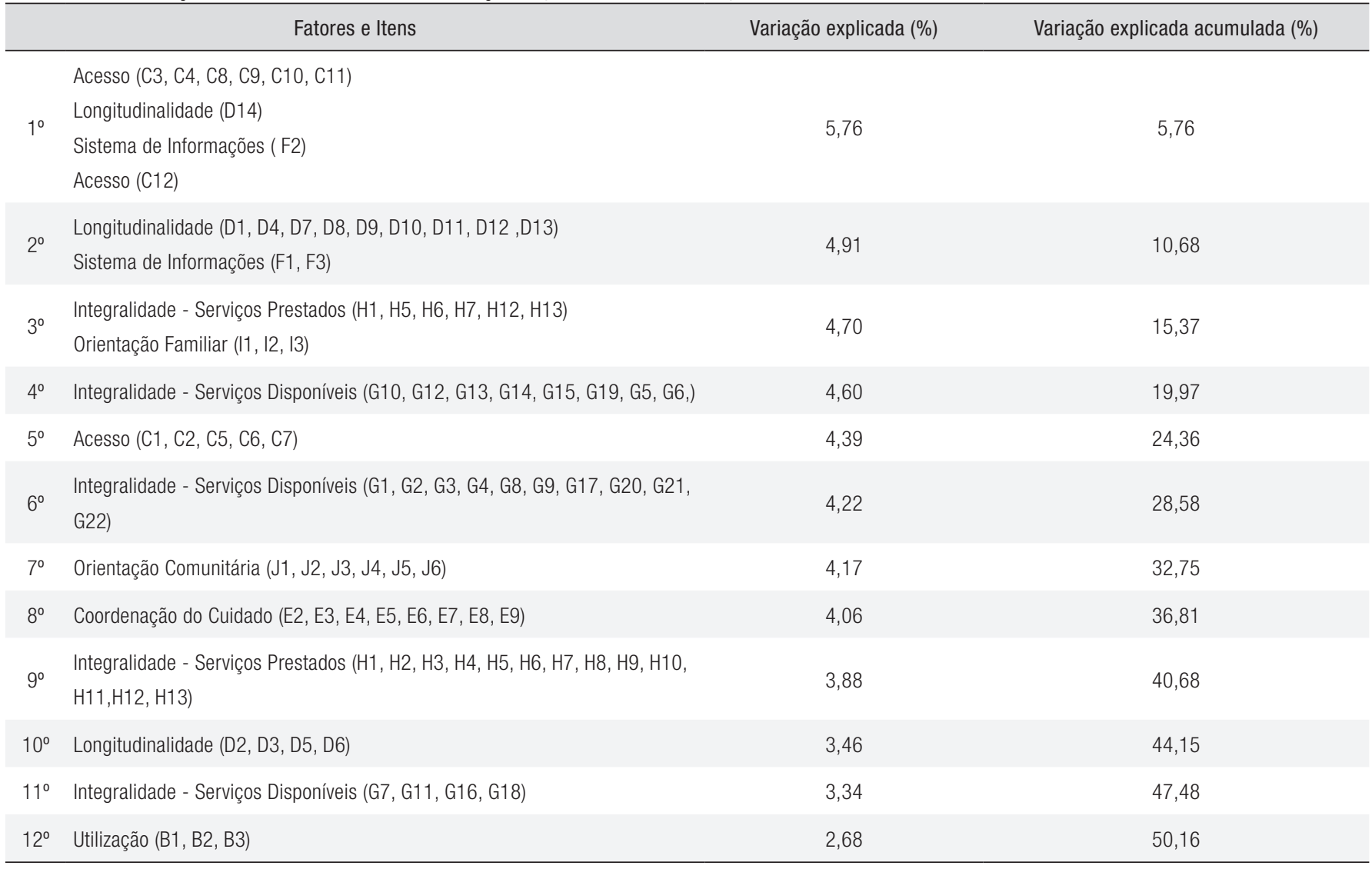

\section{Discussão}

Neste estudo foram realizadas tradução, adaptação e avaliação da validade e da fidedignidade do PCATool-Brasil versão usuários adultos, evidenciando que o instrumento é válido e fidedigno para a mensuração da qualidade dos serviços de saúde de APS a partir da experiência desses usuários. $\mathrm{Na}$ análise fatorial, o modelo com 12 fatores foi capaz de captar os quatro atributos essenciais e dois atributos derivados do conceito de APS baseado em Starfield ${ }^{3}$.

Entretanto, alguns itens não atingiram a carga fatorial mínima para a sua permanência no instrumento, como os itens que correspondem ao 'acesso no serviço de saúde até às $20 \mathrm{~h}$ em alguns dias úteis', ao 'atendimento pelo mesmo médico' e ao 'aconselhamento sobre mudanças que acontecem com o envelhecimento'. No entanto, esses itens foram, respectivamente, mantidos no componente acesso de primeiro contato, no atributo longitudinalidade e no componente integralidade de serviços disponíveis, por sua extrema relevância conceitual. Além disso, os itens que constituem o componente coordenação do sistema de informação apresentaram carga fatorial e também correlação item-total abaixo do valor recomendado para que fossem mantidos na versão brasileira do PCATool, mas também permaneceram no instrumento final exclusivamente por motivo conceitual, a fim de permitir que o instrumento validado mantivesse sua comparabilidade com o instrumento original, assim como mensurar todos os componentes dos atributos essenciais da APS.

Os resultados da "Razão de Êxito da Escala" demonstraram que os itens de cada atributo estáo bem alocados no seu atributo conceitual, pois se mostraram mais fortemente relacionados com os itens no seu componente de origem do que nos outros componentes/atributos, evidenciando, dessa maneira, a fidedignidade do PCATool. Além disso, a comparaçáo do processo de validação brasileiro com o original norte-americano ${ }^{5}$ mostra a equivalência entre os dois processos (Tabela 3).

Portanto, foram contemplados 84 itens do instrumento, que constituem seis dos sete componentes dos atributos da APS (Tabelas 3 e 4). Com o acréscimo dos três itens do componente afiliação com o serviço, tem-se o PCATool-Brasil versão usuários adultos validado com um total de 87 itens. 


\section{Conclusão}

A utilização do PCATool exige a avaliação prévia de equivalência com a versão original e a caracterização das suas propriedades psicométricas, uma vez que versôes muito distintas proporcionam dificuldades na comparação de resultados entre estudos em âmbito nacional ou internacional. O PCATool-Brasil versão usuários adultos captou os principais atributos da APS e apresentou, de modo geral, medidas de fidedignidade aceitáveis, diante da realidade na qual o instrumento foi aplicado. Portanto, mesmo considerando as diferenças regionais e o extenso número de itens, o PCATool-Brasil pode ser considerado um instrumento válido e fidedigno para avaliar a presença e extensão dos atributos da APS na experiência dos usuários adultos dos serviços de APS. Consolida-se assim como um importante instrumento para avaliar serviços de saúde, subsidiando gestores sobre a presença e extensão dos atributos da APS.

\section{Referências}

1. Brasil. Ministério da Saúde. Saúde, Brasil. 147. ed. Brasília: Ministério da Saúde; 2008.

2. Brasil. Ministério da Saúde. Atenção básica e a saúde da família: diretriz conceitual. Brasília: Ministério da Saúde; 2004.

3. Starfield B. Atenção primária: equilíbrio entre necessidades de saúde, serviços e tecnologia. Brasília: Organização das Nações Unidas para a Educação, a Ciência e a Cultura; Ministério da Saúde; 2002.

4. Cassady C, Starfield B, Hurtado M, Berk R, Nanda J, Friedenberg L. Measuring consumer experiences with primary care. J Ambulatory Pediatric Assoc. 2000; 105: 998-1003.

5. Shi L, Starfield B, Xu J. Validating the Adult Primary Care Assessment Tool. J Fam Pract 2001;50: 161W-175W.

6. Harzheim E, Starfield B, Rajmil L, Álvarez-Dardet C, Stein A. Internal consistency and reliability of Primary Care Assessment Tool (PCATool-Brasil) for child health services. Cad Saude Pública. 2006A; 22(8): 1649-59. PMid:16832536. http://dx.doi.org/10.1590/S0102-311X2006000800013

7. Harzheim E, Gonçalves MR, Oliveira MMC, Trindade TG, Agostinho MR, Hauser L. Manual do instrumento de avaliação da atenção primária à saúde: primary care assessment tool PCATool - Brasil. Brasília: Ministério da Saúde, 2010. PMCid:PMC3002662.

8. Tsai J, Shi L, Yu WL, Hung LM, Lebrun LA. Physician specialty and the quality of medical care experiences in the context of the Taiwan national health insurance system. J Am Board Fam Med. 2010; 23(3): 402-12. PMid:20453187. http://dx.doi.org/10.3122/jabfm.2010.03.090222

9. Tsai J, Shi L, Yu WL, Lebrun LA. Usual source of care and the quality of medical care experiences: a cross-sectional survey of patients from a Taiwanese community. Med Care. 2010; 48(7): 628-34. PMid:20548255. http://dx.doi.org/10.1097/MLR.0b013e3181dbdf76

10. Wong SY, Kung K, Griffiths SM, Carthy T, Wong MC, Lo SV, Chung VC, Goggins WB, Starfield B. Comparison of primary care experiences among adults in general outpatient clinics and private general practice clinics in Hong Kong. BMC Public Health. 2010; 10: 397. PMid:20602806 PMCid:PMC2908092. http://dx.doi.org/10.1186/1471-2458-10-397

11. Haggerty J, Martin CM. Evaluating Primary Health Care in Canada AND The Right Questions to Ask! The National Evaluation Strategy for Primary Health Care. Ottawa: Health Canada; 2005.

12. Haggerty JL, Pineault R, Beaulieu MD, Brunelle Y, Gauthier J, Goulet F, et al. Patients' experiences of primary care in Quebec before major reforms. Can Fam Physician. 2007; 53: 1056-1057. PMCid:PMC1949223.

13. Pasarín MI, Berra S, Rajmil L, Solans M, Borrell C, Starfield B. An instrument to evaluate primary health care from the population perspective. Aten Primaria. 2007; 39(8): 395-401. PMid:17692225.

14. Schmidt MI, Duncan BB, Silva GA, Menezes AM, Monteiro CA, Barreto SM, et al. Chronic non-communicable diseases in Brazil: burden and current challenges Lancet. 2011; 377: 1949-1961. http://dx.doi.org/10.1016/S0140-6736(11)60135-9

15. Reichenheim ME, Souza ER, Moraes CL, Jorge MHPM, Da Silva CMFP, Minayo MCS. Violence and injuries in Brazil: the effect, progress made, and challenges ahead. Lancet. 2011; 377: 1962-1975 http://dx.doi.org/10.1016/S0140-6736(11)60053-6

16. Mendes EV, As redes de atenção à saúde. Brasília: Organização Pan-Americana da Saúde, 2011.

17. Piccini RX, Facchini LA, Tomasi E, Thumé E, Silveira DS, Siqueira FV, et al. Effectiveness of antenatal and well-baby care in primary health services from Brazilian South and Northeast regions. Rev Bras Saude Mater Infant. 2007; 7(1): 75-82. http://dx.doi.org/10.1590/S1519-38292007000100009

18. Facchini LA, Piccini RX, Tomasi E, Thumé E, Silveira DS, Siqueira FV, et al. Performance of the PSF in the Brazilian South and Northeast: institutional and epidemiological Assessment of Primary Health Care. Ciênc Saúde Coletiva. 2006; 11(3): 669-681. http://dx.doi.org/10.1590/S141381232006000300015

19. Starfield B, Shi L. Manual for the Primary Care Assessment Tools. Baltimore: Johns Hopkins University, 2002.

20. Harzheim E, Duncan B, Stein A, Cunha C, Goncalves M, Trindade T, et al. Quality and effectiveness of different approaches to primary care delivery in Brazil. BMC Health Serv Res. 2006; 5(6): 156. PMid:17147819 PMCid:PMC1790713. http://dx.doi.org/10.1186/1472-6963-6-156

21. Hair JF, Anderson RE, Tatham RL, Black WC. Análise Multivariada de Dados. 5. ed. Porto Alegre: Bookman, 2006. 